\title{
Review Article \\ Input-to-State Stability of Linear Stochastic Functional Differential Equations
}

\author{
Ramazan Kadiev ${ }^{1}$ and Arcady Ponosov ${ }^{2}$ \\ ${ }^{1}$ Dagestan Research Center, The Russian Academy of Sciences and Department of Mathematics, Dagestan State University, \\ Makhachkala 367005, Russia \\ ${ }^{2}$ Department of Mathematical Sciences and Technology, Norwegian University of Life Sciences, P.O. Box 5003, 1432 Ås, Norway
}

Correspondence should be addressed to Arcady Ponosov; arkadi@nmbu.no

Received 11 February 2016; Accepted 16 March 2016

Academic Editor: Kishin Sadarangani

Copyright (C) 2016 R. Kadiev and A. Ponosov. This is an open access article distributed under the Creative Commons Attribution License, which permits unrestricted use, distribution, and reproduction in any medium, provided the original work is properly cited.

\begin{abstract}
The purpose of the paper is to show how asymptotic properties, first of all stochastic Lyapunov stability, of linear stochastic functional differential equations can be studied via the property of solvability of the equation in certain pairs of spaces of stochastic processes, the property which we call input-to-state stability with respect to these spaces. Input-to-state stability and hence the desired asymptotic properties can be effectively verified by means of a special regularization, also known as "the $W$-method" in the literature. How this framework provides verifiable conditions of different kinds of stochastic stability is shown.
\end{abstract}

\section{Introduction}

This review paper is aimed at describing a general framework for analysis of asymptotic properties of linear stochastic functional differential equation driven by a semimartingale. The core idea of the method is an alternative description of asymptotic properties in terms of solvability of the equation in certain pairs of spaces of stochastic processes on the semiaxis. Similar to the deterministic case, this property can be called input-to-state stability (ISS) [1] or, alternatively, admissibility of the pairs of spaces for the equation in question $[2,3]$.

As long as the relationship between a desired asymptotic property and ISS with respect to a certain pair of spaces is established, one applies a special regularization method to verify ISS. Usually, such a regularization starts with choosing a simpler equation (called a reference equation), which is already ISS with respect to this pair of spaces. Resolved and substituted into the original equation, the reference equation produces a new, integral equation of the form $x-\Theta x=f$. If the latter is solvable (e.g., if $\|\Theta\|<1$ ) in a suitable space, then ISS and hence the related asymptotic property are proved.

This framework was proposed by Azbelev (who also gave the name the $W$-method to this framework) and his students in 1986 for stability analysis of deterministic functional differential equations. The $W$-method was meant to serve as an alternative to the Lyapunov direct method for linear delay equations (see [4]). Later on the method was generalized in $[2,5-7]$ (see also the references therein) and applied to other classes of equations, for example, in [8-10] and in many other papers. In [3], the method was for the first time applied to linear stochastic functional differential equations and developed further in [11-25]. In the recent paper [26] the idea of input-to-state stability was applied to nonlinear stochastic equations describing neural networks.

In some sense, the $W$-method is similar to Lyapunov's direct method. But instead of seeking a Lyapunov function(al) one aims to find a suitable reference equation which possesses the prescribed ISS property and which then is used to regularize the original equation. Like Lyapunov's method, the $W$-method also provides necessary and sufficient stability conditions (currently for linear equations, only).

The present review paper offers a short yet consistent description of the results which have been published by the authors since 1992. The material is organized as follows. Notation and a short introduction to the concept of a linear stochastic functional differential equation are given in Section 2. In Section 3 we introduce (stochastic) input-to-state 
stability, describe its connections to various types of stochastic Lyapunov stability, and outline two regularization methods (left and right $W$-transforms). In Section 4 we provide conditions which guarantee ISS in the weighted spaces (BohlPerron type theorems). These results are used to deduce asymptotic Lyapunov stability from simple stability. Applications to stochastic functional differential and difference equations are discussed in Sections 5 and 6, respectively. These two chapters contain several concrete examples of stochastic delay equations, which demonstrate efficiency of our method. Finally, in Section 7 we offer a short summary and mention several generalizations of the results presented.

Most proofs, many of which are rather technical, are omitted. In such a case, the papers are cited right before the corresponding theorems, where detailed proofs are available.

\section{Preliminaries and the Concept of a Stochastic Functional Differential Equation}

Let $\left(\Omega, \mathscr{F},(\mathscr{F})_{t \geq 0}, P\right)$ be a stochastic basis (see, e.g., [27]), where $\Omega$ is a set of elementary probability events, $\mathscr{F}$ is a $\sigma$ algebra of all events on $\Omega,(\mathscr{F})_{t \geq 0}$ is a right continuous family of $\sigma$-subalgebras of $\mathscr{F}$, and $P$ is a probability measure on $\mathscr{F}$; all the above $\sigma$-algebras are assumed to be complete with respect to the measure $P$, that is, containing all subsets of zero measure; the symbol $E$ stands below for the expectation related to the probability measure $P$.

In the sequel, we will use an arbitrary yet fixed norm $|\cdot|$ in $R^{n},\|\cdot\|$ being the associated matrix norm.

$\bar{E}$ stands for the identity matrix (as long as its size is defined).

The space $k^{n}$ consists of all $n$-dimensional, $\mathscr{F}_{0}$-measurable random variables, and $k=k^{1}$ is a commutative ring of all scalar $\mathscr{F}_{0}$-measurable random variables.

By $Z:=\left(z_{1}, \ldots, z_{m}\right)^{T}$ we denote an $m$-dimensional semimartingale (see, e.g., [27]), while $\mathscr{B}_{i}, i=2, \ldots, m$, will be mutually independent standard Brownian motions (Wiener processes). tion

We consider a homogeneous stochastic hereditary equa-

$$
d x(t)=\left(V_{h} x\right)(t) d Z(t) \quad(t \geq 0)
$$

equipped with two extra conditions

$$
\begin{aligned}
& x(s)=\varphi(s) \quad(s<0), \\
& x(0)=x_{0} .
\end{aligned}
$$

Here $V_{h}$ is a $k$-linear Volterra operator (see the following), which is defined in certain linear spaces of vector stochastic processes, $\varphi$ is an $\mathscr{F}_{0}$-measurable stochastic process, and $x_{0} \in$ $k^{n}$.

By $k$-linearity of the operator $V_{h}$ we mean the following property:

$$
V_{h}\left(\alpha_{1} x_{1}+\alpha_{2} x_{2}\right)=\alpha_{1} V_{h} x_{1}+\alpha_{2} V_{h} x_{2}
$$

holding for all $\mathscr{F}_{0}$-measurable, bounded, and scalar random values $\alpha_{1}, \alpha_{2}$ and all stochastic processes $x_{1}, x_{2}$ belonging to the domain of the operator $V_{h}$.
The solution of the initial value problem $((1)-(2 \mathrm{~b}))$ will be denoted by $x\left(t, x_{0}, \varphi\right), t \in(-\infty, \infty)$. Below the solution is always assumed to exist and be unique for an appropriate choice of $\varphi(s), x_{0}$ : for specific conditions see, for example, [13, 28].

The following kinds of stochastic Lyapunov stability will be discussed in this paper.

Definition 1. For a given real number $p(0<p<\infty)$ one calls the zero solution of the homogeneous equation (1)

(i) $p$-stable (with respect to the initial data, i.e., with respect to $x_{0}$ and the "prehistory" function $\varphi$ ) if for any $\epsilon>0$ there is $\delta(\epsilon)>0$ such that $E\left|x_{0}\right|^{p}+$

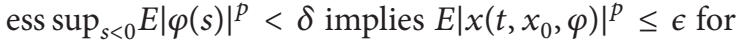
all $t \geq 0$ and all (admissible) $\varphi, x_{0}$;

(ii) asymptotically $p$-stable (with respect to the initial data) if it is $p$-stable and, in addition, any $\varphi, x_{0}$

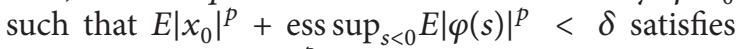
$\lim _{t \rightarrow+\infty} E\left|x\left(t, x_{0}, \varphi\right)\right|^{p}=0$;

(iii) exponentially $p$-stable (with respect to the initial data) if there exist positive constants $\bar{c}, \beta$ such that the inequality $E\left|x\left(t, x_{0}, \varphi\right)\right|^{p} \leq \bar{c}\left(E\left|x_{0}\right|^{p}+\right.$

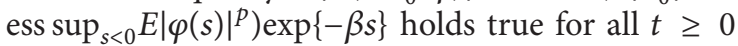
and all $\varphi, x_{0}$.

Remark 2. If (1) is an ordinary differential equation, then Definition 1 converts into the well-known definition of the moment stability with respect to the random initial values from $k^{n}$.

To be able to link stochastic Lyapunov stability to ISS, we need to represent (1), (2a), and (2b) in a different manner. Let $x(t)$ be a stochastic process on the real semiaxis $(t \in[0,+\infty))$ and $x_{+}(t)$ a stochastic process on the entire real axis $(t \in$ $(-\infty,+\infty))$ coinciding with $x(t)$ for $t \geq 0$ and equalling 0 for $t<0$, and let $\varphi_{-}(t)$ be a stochastic process on the axis $(t \in(-\infty,+\infty))$ coinciding with $\varphi(t)$ for $t<0$ and equalling 0 for $t \geq 0$. Then the stochastic process $x_{+}(t)+\varphi_{-}(t)$ defined for $t \in(-\infty,+\infty)$ will be a solution of problem (1), (2a), and (2b) if $x(t)(t \in[0,+\infty))$ satisfies the initial value problem

$$
\begin{aligned}
d x(t) & =[(V x)(t)+f(t)] d Z(t) \quad(t \geq 0), \\
x(0) & =x_{0},
\end{aligned}
$$

where $(V x)(t):=\left(V_{h} x_{+}\right)(t)$ and $f(t):=\left(V_{h} \varphi_{-}\right)(t)$ for $t \geq$ 0 . Indeed, by linearity $V_{h}\left(x_{+}+\varphi_{-}\right)=V_{h}\left(x_{+}\right)+V_{h}\left(\varphi_{-}\right)=$ $V x+f$, which gives (4). Note that $f$ is uniquely defined by the stochastic process $\varphi$, "the prehistory function." Let us also observe that the initial value problem (4) and (5a) is equivalent to the initial value problem (1), (2a), and (2b) only for $f$, which have representation $f=V_{h} \varphi^{\prime}$, where $\varphi^{\prime}$ is an arbitrary extension of the function $\varphi$ to the real axis $(-\infty, \infty)$.

In the sequel the following linear spaces of stochastic processes will be used:

(i) $L^{n}(Z)$ consists of all predictable $n \times m$-matrix stochastic processes on $[0,+\infty)$, the rows of which are locally integrable with respect to the semimartingale $Z$ (see, e.g., [29] or [27]). 
(ii) $D^{n}$ consists of all $n$-dimensional stochastic processes on $[0,+\infty)$, which can be represented as

$$
x(t)=x(0)+\int_{0}^{t} H(s) d Z(s)
$$

where $x(0) \in k^{n}, H \in L^{n}(Z)$.

Let $B$ be a linear subspace of the space $L^{n}(Z)$ equipped with some norm $\|\cdot\|_{B}$. For a given positive and continuous function $\gamma(t)(t \in[0, \infty))$ we define $B^{\gamma}=\{f: f \in B, \gamma f \in$ $B\}$. The latter space becomes a linear normed space if we put $\|f\|_{B^{\nu}}:=\|\gamma f\|_{B^{\prime}}$.

We will also need the following linear subspaces of "the space of initial values" $k^{n}$ and "the space of solutions" $D^{n}$ :

$$
\begin{aligned}
k_{p}^{n} & =\left\{\alpha: \alpha \in k^{n}, E|\alpha|^{p}<\infty\right\} ; \\
M_{p}^{\gamma} & =\left\{x: x \in D^{n}, \sup _{t \geq 0} E|\gamma(t) x(t)|^{p}<\infty\right\}, \\
M_{p}^{1} & =M_{p} .
\end{aligned}
$$

For $1 \leq p<\infty$ the linear spaces $k_{p}^{n}, M_{p}^{\gamma}$ become normed spaces if we define $\|\alpha\|_{k_{p}^{n}}=\left(E|\alpha|^{p}\right)^{1 / p}$ and $\|x\|_{M_{p}^{\gamma}}=$ $\sup _{t \geq 0}\left(E|\gamma(t) x(t)|^{p}\right)^{1 / p}$.

The main focus of the present paper is put on (4), where $V: D^{n} \rightarrow L^{n}(Z)$ is a $k$-linear Volterra operator and $f \in$ $L^{n}(Z)$. Recall that $V: D^{n} \rightarrow L^{n}(Z)$ is said to be Volterra if, for any (random) stopping time $\tau, \tau \in[0,+\infty)$ a.s. and for any stochastic processes $x, y \in D^{n}$ the equality $x(t)=y(t)$ $(t \in[0, \tau]$ a.s. $)$ implies the equality $(V x)(t)=(V y)(t)(t \in$ $[0, \tau]$ a.s.).

In the sequel, we will always assume that the operator $V$ : $D^{n} \rightarrow L^{n}(Z)$ in (4), (5a) is a $k$-linear Volterra operator and $f \in L^{n}(Z)$ and $x_{0} \in k^{n}$.

A solution of the initial value problem (4) and (5a) is a stochastic process from the space $D^{n}$ satisfying the equation

$$
x(t)=x_{0}+(F x)(t) \quad(t \geq 0),
$$

where $(F x)(t)=\int_{0}^{t}[(V x)(s)+f(s)] d Z(s)$ is a $k$-linear Volterra operator in the space $D^{n}$ and the integral is understood as a stochastic one with respect to the semimartingale $Z$ (see, e.g., [27] or [29]) and $x_{0} \in k^{n}$.

Equation (4) will be referred to as a linear functional differential equation with respect to a semimartingale. For $Z(t)=\operatorname{col}\left(t, \mathscr{B}_{2}(t), \ldots, \mathscr{B}_{m}(t)\right)$ we obtain a linear functional differential equation of Itô type which is a particular case of the general equation (4).

According to [3] (see also the habilitation thesis [13]) the following classes of linear stochastic equations also are particular cases of (4):

(a) Systems of linear ordinary (i.e., nondelay) stochastic differential equations driven by an arbitrary semimartingale (in particular, systems of ordinary Itô equations). (b) Systems of linear stochastic differential equations with discrete delays driven by a semimartingale (in particular, systems of Itô equations with discrete delays).

(c) Systems of linear stochastic differential equations with distributed delays driven by a semimartingale (in particular, systems of Itô equations with distributed delays).

(d) Systems of linear stochastic integrodifferential equations driven by a semimartingale (in particular, systems of Itô integrodifferential equations).

(e) Systems of linear stochastic functional difference equations driven by a semimartingale (in particular, systems of Itô functional difference equations).

For instance, a general linear stochastic differential equation with distributed delay

$$
d x(t)=\left(V_{h} x\right)(t) d Z(t) \quad(t \geq 0),
$$

where

$$
\begin{gathered}
\left(V_{h} x\right)(t)=\left(\int_{(-\infty, t)} d_{s} \mathscr{R}_{1}(t, s) x(s), \ldots,\right. \\
\left.\int_{(-\infty, t)} d_{s} \mathscr{R}_{m}(t, s) x(s)\right)
\end{gathered}
$$

and $\mathscr{R}_{i}$ are vector functions defined on $\{(t, s): t \in[0, \infty)$, $-\infty<s \leq t\}$ for $i=1, \ldots, m$, which is equipped with the prehistory condition

$$
x(\nu)=\varphi(\nu) \quad(\nu<0),
$$

can be, under natural assumptions on $\mathscr{R}_{i}$ and $\varphi$ (see, e.g., [3, 13]), rewritten as the functional differential equation (4) with

$$
\begin{gathered}
(V x)(t)=\left(\int_{[0, t)} d_{s} \mathscr{R}_{1}(t, s) x(s), \ldots,\right. \\
\left.\int_{[0, t)} d_{s} \mathscr{R}_{m}(t, s) x(s)\right) \\
f(t)=\left(\int_{(-\infty, 0)} d_{s} \mathscr{R}_{1}(t, s) \varphi(s), \ldots\right. \\
\left.\int_{(-\infty, 0)} d_{s} \mathscr{R}_{m}(t, s) \varphi(s)\right)
\end{gathered}
$$

It is also worth mentioning that our concept of a stochastic functional differential equation covers as well the case of functional differential equations with respect to random Borel measures. In this case, we can simply put $Z(t)=\lambda(t)$ in (4), where $\lambda(t)$ is a random Borel measure of bounded variation, so that the space $L^{n}(Z)$ contains all $n$-dimensional stochastic processes on $[0,+\infty)$ with the trajectories that are a.s. locally integrable with respect to $\lambda$. Thus, (4) contains linear random differential equations including no delay, discrete and distributed delays, and linear random integrodifferential equations and linear random functional difference equations. Finally, if $\lambda(t)$ is nonrandom (or equivalently if $\Omega$ contains only one point), then we obtain the deterministic versions of all the above classes of equations. 


\section{Input-to-State Stability}

Below $x_{f}\left(t, x_{0}\right)$ stands for the solution of the initial value problem (4) and (5a), that is, for the stochastic process satisfying (4) and the initial condition $(5 \mathrm{a}): x_{f}\left(0, x_{0}\right)=x_{0}$. In the case of the homogeneous equation (4) we will also write $x\left(t, x_{0}\right)=x_{0}\left(t, x_{0}\right)$.

Representation of solutions of the deterministic functional differential equations (the generalized Cauchy formula) plays an important role in the stability analysis and in the theory of quasilinear equations. The following lemma gives the representation of the solutions of the initial value problem (4) and (5a).

Lemma 3. Let the initial value problem (4) and (5a) have a unique (up to the P-equivalence) solution $x_{f}\left(t, x_{0}\right)$ for any $f \in$ $L^{n}(Z)$ and $x_{0} \in k^{n}$. Then one has the following representation:

$$
x_{f}\left(t, x_{0}\right)=X(t) x_{0}+(C f)(t) \quad(t \geq 0)
$$

where $X(t)(X(0)=\bar{E})$ is an $n \times n$-matrix, the columns of which satisfy the homogeneous equation corresponding to (4) (the fundamental matrix) and $C: L^{n}(Z) \rightarrow D^{n}$ is a $k$-linear operator (the Cauchy or Green's operator), for which $(C f)(0)=$ 0 and $C f$ is a solution of (4).

Proof. Using the $k$-linearity of the operator $V$ it is easy to see that $X(t) x_{0}$ is a solution of the homogeneous equation corresponding to (4). Now consider

$$
x(0)=0 \text {. }
$$

Due to the assumptions of the lemma, the initial value problem (4) and (13b) has a unique solution for any $f \in$ $L^{n}(Z)$. Thus, this problem gives rise to an operator from $L^{n}(Z)$ to $D^{n}$. Denote this operator by $C$. Clearly, $(C f)(0)=0$. The $k$-linearity of $C$ follows directly from the $k$-linearity of the operator $V$ and the unique solvability of the initial value problem (4) and (13b). Therefore, the stochastic process on the right-hand side of (13) satisfies (4) and (5a).

Remark 4. For some classes of (4), an explicit integral formula for the operator $C$ in the representation (13) can be given. The corresponding results can be found in [13]. The problems of existence and uniqueness of solutions of the initial value problem (4) and (5a) are considered in $[13,28]$.

Definition 5. Let $1 \leq p<\infty$. One says that (4) is inputto-state stable (ISS) with respect to the pair $\left(M_{p}^{\gamma}, B^{\gamma}\right)$ if there exists $\bar{c}>0$, for which $x_{0} \in k_{p}^{n}$ and $f \in B^{\gamma}$ imply the relation $x_{f}\left(\cdot, x_{0}\right) \in M_{p}^{\gamma}$ and the following estimate:

$$
\left\|x_{f}\left(\cdot, x_{0}\right)\right\|_{M_{p}^{\gamma}} \leq \bar{c}\left(\left\|x_{0}\right\|_{k_{p}^{n}}+\|f\|_{B^{\gamma}}\right) \text {. }
$$

This definition says that the solutions belong to $M_{p}^{\gamma}$ whenever $f \in B^{\gamma}$ and $x_{0} \in k_{p}^{n}$ and that they continuously depend on $f$ and $x_{0}$ in the appropriate topologies. The choice of the spaces is closely related to the kind of stability we are interested in. Formally speaking, one has to mention the space of initial values $k_{p}^{n}$ in this definition [25]. However, this space is kept fixed in the present paper, so that we skip it.

The following result proved in $[3,13]$ uncovers connection between stability of the zero solution of (1) and (2a) and the admissibility of spaces for (4) with the operator $V$ which is constructed from the operator $V_{h}$ in (1) and (2a) in the manner described at the beginning of Section 2.

Theorem 6. Let $\gamma(t)(t \geq 0)$ be a positive continuous function and $1 \leq p<\infty$. Assume that (4) is constructed from (1) and (2a) in the manner described in Section 2 and that $f(t) \equiv\left(V_{h} \varphi_{-}\right)(t) \in B^{\gamma}$ whenever $\varphi$ satisfies the condition

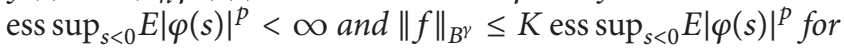
some constant $K>0$.

(1) If $\gamma(t)=1(t \geq 0)$ and (4) is ISS with respect to the pair $\left(M_{p}^{\gamma}, B^{\gamma}\right)$, then the zero solution of (1) is p-stable.

(2) If $\gamma(t)=\exp \{\beta t\}(t \geq 0)$ for some $\beta>0$ and (4) is ISS with respect to the pair $\left(M_{p}^{\gamma}, B^{\gamma}\right)$, then the zero solution of (1) is exponentially p-stable.

(3) If $\lim _{t \rightarrow+\infty} \gamma(t)=+\infty, \gamma(t) \geq \delta>0, t \in[0,+\infty)(t \geq$ $0)$ for some $\delta$, and (4) is ISS with respect to the pair $\left(M_{p}^{\gamma}, B^{\gamma}\right)$, then the zero solution of $(1)$ is asymptotically p-stable.

Below we demonstrate how this result leads to efficient algorithms in analysis of Lyapunov stability of linear stochastic functional differential equations. The main idea, which is described in $[3,19-21]$, is to convert the given property of Lyapunov stability, via the property of ISS, into the property of invertibility of a certain regularized operator in a suitable functional space. This operator can be constructed with the help of an auxiliary equation.

The description of this algorithm applied to (4) starts from choosing an auxiliary equation, which we call $a$ reference equation. The latter is similar to (4), but it is "simpler," so that the required ISS property is already established for this equation:

$$
d x(t)=[(Q x)(t)+g(t)] d Z(t) \quad(t \geq 0),
$$

where $Q: D^{n} \rightarrow L^{n}(Z)$ is a $k$-linear Volterra operator and $g \in L^{n}(Z)$. For (15) the existence and uniqueness property is always assumed, that is, that for any $x(0) \in k^{n}$ there is the only (up to a $P$-equivalence) solution $x(t)$ satisfying (15). According to the lemma, for the solutions of (15) we have the following representation:

$$
x(t)=U(t) x_{0}+(W g)(t) \quad(t \geq 0),
$$

where $U(t)$ is the fundamental matrix of the associated homogeneous equation and $W$ is the corresponding Cauchy operator for (15).

As in the deterministic case, we have two versions of the regularization: the right and the left one. They stem formally from the same reference equation but produce different integral equations. Also the applicability conditions are different. 
We start with the right regularization.

Inserting (16a) into (4) yields

$$
\begin{aligned}
& {[(Q U x(0))(t)+(Q W g)(t)+g(t)] d Z(t)} \\
& \quad=[(V(U x(0)+W g))(t)+f(t)] d Z(t) \quad(t \geq 0) .
\end{aligned}
$$

Denoting $(V-Q) W=\Theta_{r}$, we obtain the operator equation

(1)

$$
\left(I-\Theta_{r}\right) g=(V-Q) U x(0)+f .
$$

The regularization is called "right" as the operator $W$ is placed to the right of the operator $V$ in (4). The letter " $r$ " in the operator $\Theta_{r}$ is due to the word "right."

The next result of this section lists the assumptions on the reference equation, under which the right regularization may be applied.

Theorem 7. Given a weight $\gamma$ (i.e., a positive continuous function defined for $t \geq 0$ ), let one assume that (4) and the reference equation (15) satisfy the following conditions:

(1) The operators $V$ and $Q$ act continuously from $M_{p}^{\gamma}$ to $B^{\gamma}$.

(2) The reference equation (15) is ISS with respect to the $\operatorname{pair}\left(M_{p}^{\gamma}, B^{\gamma}\right)$.

If now the operator $I-\Theta_{r}: B^{\gamma} \rightarrow B^{\gamma}$ has a bounded inverse, then (4) is ISS with respect to the pair $\left(M_{p}^{\gamma}, B^{\gamma}\right)$.

Proof. Under the assumptions of the theorem we have

$$
\begin{aligned}
x_{f}\left(t, x_{0}\right)= & U(t) x_{0} \\
& +\left(W\left(I-\Theta_{r}\right)^{-1}(V-Q) U x_{0}\right)(t) \\
& +\left(W\left(I-\Theta_{r}\right)^{-1} f\right)(t)
\end{aligned}
$$

for arbitrary $x_{0} \in k_{p}^{n}, f \in B^{\gamma}$. The ISS property of the reference equation implies

$$
\begin{array}{r}
\left\|U(t) x_{0}\right\|_{M_{p}^{\gamma}} \leq \widetilde{c}\left\|x_{0}\right\|_{k_{p}^{n}}, \\
\|W g\|_{M_{p}^{\gamma}} \leq \widetilde{c}\|g\|_{B^{\gamma}},
\end{array}
$$

which holds for all $x_{0} \in k_{p}^{n}, g \in B^{\gamma}$. Here $\widetilde{c}$ is some positive number. Taking now the norms we arrive at the inequality

$$
\left\|x_{f}\left(\cdot, x_{0}\right)\right\|_{M_{p}^{\gamma}} \leq \bar{c}\left(\left\|x_{0}\right\|_{k_{p}^{n}}+\|f\|_{B^{\gamma}}\right)
$$

for some $\bar{c}>0$. This implies that (4) is ISS with respect to the pair $\left(M_{p}^{\gamma}, B^{\gamma}\right)$.

Consider now the case of the left regularization rewriting (4) as follows:

$$
d x(t)=[(Q x)(t)+((V-Q) x)(t)+f(t)] d Z(t)
$$

$$
(t \geq 0) \text {, }
$$

or alternatively as

$$
\begin{array}{r}
x(t)=U(t) x(0)+(W(V-Q) x)(t)+(W f)(t) \\
(t \geq 0) .
\end{array}
$$

Denoting $W(V-Q)=\Theta_{l}$, we obtain the operator equation (2)

$$
\left(\left(I-\Theta_{l}\right) x\right)(t)=U(t) x(0)+(W f)(t) \quad(t \geq 0) .
$$

Theorem 8. Given a weight $\gamma$ (i.e., a positive continuous function defined for $t \geq 0$ ), let one assume that (4) and the reference equation (15) satisfy the following conditions:

(1) The operators $V$ and $Q$ act continuously from $M_{p}^{\gamma}$ to $B^{\gamma}$.

(2) The reference equation (15) is ISS with respect to the $\operatorname{pair}\left(M_{p}^{\gamma}, B^{\gamma}\right)$.

If now the operator $I-\Theta_{l}: M_{p}^{\gamma} \rightarrow M_{p}^{\gamma}$ has a bounded inverse in this space, then (4) is ISS with respect to the pair $\left(M_{p}^{\gamma}, B^{\gamma}\right)$.

Proof. Under the above assumptions we have that $U(\cdot) x_{0} \in$ $M_{p}^{\gamma}$ whenever $x_{0} \in k_{p}^{n}$ and also that

$$
\begin{aligned}
x_{f}\left(t, x_{0}\right)= & \left(\left(I-\Theta_{l}\right)^{-1}\left(U(\cdot) x_{0}\right)\right)(t) \\
& +\left(\left(I-\Theta_{l}\right)^{-1} W f\right)(t) \quad(t \geq 0)
\end{aligned}
$$

for arbitrary $x_{0} \in k_{p}^{n}, f \in B^{\gamma}$. Taking the norms and using the assumptions put on the reference equation, we, as in the previous theorem, obtain the inequality

$$
\left\|x_{f}\left(\cdot, x_{0}\right)\right\|_{M_{p}^{\gamma}} \leq \bar{c}\left(\left\|x_{0}\right\|_{k_{p}^{n}}+\|f\|_{B^{\gamma}}\right),
$$

where $x_{0} \in k_{p}^{n}, f \in B^{\gamma}$. Thus, (4) is ISS with respect to the pair $\left(M_{p}^{\gamma}, B^{\gamma}\right)$.

The left and right regularization give usually different stability results, in both the deterministic and stochastic theory. In the stochastic case, the left regularization appears to be more efficient.

Finally, we remark that the choice of the space $B$ and the weight $\gamma$ depends on the asymptotic property we are studying. In the next section we describe typical examples which are general enough to cover most interesting cases of stochastic stability and, on the other hand, specific enough to ensure applicability of the important Bohl-Perron property.

\section{ISS with respect to Weighted Spaces and Bohl-Perron Type Theorems}

By Bohl-Perron type theorems one means results ensuring equivalence between ISS with respect to the spaces with and without weights. This allows for deducing asymptotic (exponential) stability from the simple stability which is much easier to handle.

For technical reasons we restrict ourselves to the so-called special semimartingales in this section. In this case we will be 
able to give a more explicit description of the spaces $D^{n}$ and $L^{n}(Z)$.

A special semimartingale $Z(t)(t \in[0, \infty))$ can be represented as a sum

(3)

$$
Z(t)=b(t)+c(t) \quad(t \geq 0)
$$

where $b(t)$ is a predictable stochastic process of locally finite variation and $c(t)$ is a local square-integrable martingale [27, p. 28] such that all the components of the process $b(t)$ as well as the predictable characteristics $\left\langle c^{i}, c^{j}\right\rangle(t), 1 \leq i, j \leq m$, of the process $c(t)[27$, p. 48] are absolutely continuous with respect to a nondecreasing function $\lambda:[0, \infty) \rightarrow \mathbf{R}_{+}$. Under these assumptions we can write

$$
\begin{aligned}
b^{i} & =\int_{0} a^{i} d \lambda, \\
\left\langle c^{i}, c^{j}\right\rangle & =\int_{0}^{\cdot} A^{i j} d \lambda,
\end{aligned}
$$

$$
i, j=1, \ldots, m
$$

(e.g., $\lambda(t)=t$ for Itô equations). Without loss of generality, it is convenient to assume that the first component of the semimartingale $Z(t)$ coincides with $\lambda(t)$; that is, $z^{1}(t)=\lambda(t)$. Clearly, we can always do it by adding, if necessary, new, $(m+1)$ th component to the $m$-dimensional semimartingale $Z(t)$.

It is known [29] that for special semimartingales the space $L^{n}(Z)$ consists of all predictable $n \times m$-matrices $H(t)=$ $\left[H^{i j}(t)\right]$, for which

$$
\int_{0}^{t}\left(|H a|+\left\|H A H^{\top}\right\|\right) d \lambda<\infty \text { a.s. }
$$

for any $t \geq 0$. Here

$$
\begin{aligned}
& a:=\left(a^{1}, \ldots, a^{m}\right)^{T}, \\
& A:=\left[A^{i j}\right] .
\end{aligned}
$$

Under the above assumptions we can also write $\int_{0}^{t} H d Z=\int_{0}^{t} H d b+\int_{0}^{t} H d c$.

Finally, the space $D^{n}$ consists of all $n$-dimensional $\mathscr{F}_{t}$-adapted stochastic processes on $[0,+\infty)$, which are right continuous and have left-hand side limits at all points.

The following particular cases of the general space $B$ are crucial for our further considerations:

$$
\begin{aligned}
& \Lambda_{p, q}^{n}\left(\xi, K_{1}, K_{2}\right)=\{H: H \\
& \quad \in L^{n}(Z),\left(E|H a|^{p}\right)^{1 / p} \xi^{1 / q-1} \\
& \left.\quad \in L_{q}^{\lambda},\left(E\left\|H A H^{\top}\right\|^{p / 2}\right)^{1 / p} \xi^{1 / q-1 / 2} \in L_{q}^{\lambda}\right\},
\end{aligned}
$$

$$
\begin{aligned}
& \|H\|_{\Lambda_{p, q}^{n}\left(\xi, K_{1}, K_{2}\right)} \stackrel{\text { def }}{=}\left\|\left(E\left|K_{1} H a\right|^{p}\right)^{1 / p} \xi^{(1 / q-1)}\right\|_{L_{q}^{\lambda}} \\
& +\left\|\left(E\left\|K_{2} H A H^{\top}\right\|^{p / 2}\right)^{1 / p} \xi^{1 / q-1 / 2}\right\|_{L_{q}^{\lambda}} ; \\
& \Lambda_{p, q}^{n+}\left(\xi, K_{1}, K_{2}\right)=\{H: H \\
& \in L^{n}(Z),\left(E\left|H^{+} a^{+}\right|^{p}\right)^{1 / p} \xi^{1 / q-1} \\
& \left.\in L_{q}^{\lambda},\left(E\left\|H^{+} A^{+}\left(H^{+}\right)^{\top}\right\|^{p / 2}\right)^{1 / p} \xi^{1 / q-1 / 2} \in L_{q}^{\lambda}\right\}, \\
& \|H\|_{\Lambda_{p, q}^{n+}\left(\xi, K_{1}, K_{2}\right)} \stackrel{\text { def }}{=}\left\|\left(E\left|K_{1} H^{+} a^{+}\right|^{p}\right)^{1 / p} \xi^{(1 / q-1)}\right\|_{L_{q}^{\lambda}} \\
& +\left\|\left(E\left\|K_{2} H^{+} A^{+}\left(H^{+}\right)^{\top}\right\|^{p / 2}\right)^{1 / p} \xi^{1 / q-1 / 2}\right\|_{L_{q}^{\lambda}} .
\end{aligned}
$$

The following parameters are used in the above definitions:

(i) The function $\xi$ is a nonnegative measurable function defined for $t \geq 0$.

(ii) $K_{1}, K_{2}$ are two positive real numbers.

(iii) The real numbers $p, q$ satisfy the inequalities $1 \leq p<$ $\infty, 1 \leq q \leq \infty$.

(iv) $a^{+}, A^{+}, h^{+}$are defined in the same way as $a$ and $A$ above, namely, by the equalities

$$
\begin{aligned}
a^{+} & :=\left(\left|a^{1}\right|, \ldots,\left|a^{m}\right|\right)^{T}, \\
A^{+} & :=\left[\left|A^{i j}\right|\right], \\
h^{+} & :=\left[\left|h^{j}\right|\right] .
\end{aligned}
$$

(v) $L_{q}^{\lambda}$ is the linear space of scalar functions defined on $[0,+\infty), q$-integrable $(1 \leq q<\infty)$ with respect to the increasing function $\lambda$, and essentially (with respect to the measure generated by $\lambda$ ) bounded if $q=\infty$.

Notice that the spaces $\Lambda_{p, q}^{n}\left(\xi, K_{1}, K_{2}\right)$ consist of the same stochastic processes; that is, they in fact are independent of the choice of the positive numbers $K_{1}, K_{2}$, the only difference being the norms in these spaces, which is introduced by technical reasons. The same applies to the spaces $\Lambda_{p, q}^{+}\left(\xi, K_{1}, K_{2}\right)$. We remark that all of these spaces are of importance for analysis of ISS of (4).

In the sequel we will also write $\Lambda_{p, q}^{n}(\xi)$ instead of $\Lambda_{p, q}^{n}(\xi, 1,1)$ and $\Lambda_{p, q}^{+}(\xi)$ instead of $\Lambda_{p, q}^{+}(\xi, 1,1)$.

In the rest of the section we assume that $\gamma(t)=$ $\exp \left(\beta \int_{0}^{t} \xi(s) d \lambda(s)\right)(t \geq 0)$, where $\xi$ is a scalar, nonnegative function $\xi$, defined on $[0, \infty)$ and integrable with respect to the measure generated by the function $\lambda$. 
We assume now that the $k$-linear operator $V$ in (4) acts as follows:

$$
V: M_{p} \longrightarrow \Lambda_{p, q}^{n}\left(\xi, K_{1}, K_{2}\right)
$$

(for some admissible $p, q, \xi, K_{1}, K_{2}$ ). We will also use the following notation related to the operator $V$ :

(i) $V x=\left(V_{1} x, \ldots, V_{m} x\right)$;

(ii) $\left(V^{\beta} x\right)(t):=\gamma(t)(V(x / \gamma))(t)$, where $\gamma(t)$.

Finally, we let $m_{p}$ stand for the space $M_{p}$ in the scalar case.

Definition 9. One says that a $k$-linear Volterra operator $\bar{V}$ : $m_{p} \rightarrow \Lambda_{p, q}^{1+}\left(\xi, K_{1}, K_{2}\right)$ dominates a $k$-linear Volterra operator $V: M_{p} \rightarrow \Lambda_{p, q}^{n}\left(\xi, K_{1}, K_{2}\right)$, if (1) $\bar{V}$ is positive, that is, $x \geq 0$ a.s. implies $\bar{V} x \geq 0$ a.s., and (2) $\left(\left|V_{1} x\right|, \ldots,\left|V_{m} x\right|\right) \leq \bar{V}|x|$ a.s. for any $x \in M_{p}$.

Definition 10. One says that a $k$-linear Volterra operator $V$ : $M_{p} \rightarrow \Lambda_{p, q}^{n}\left(\xi, K_{1}, K_{2}\right)$ satisfies the $\Delta$-condition if $V$ is dominated by some $k$-linear Volterra operator $\bar{V}: m_{p} \rightarrow \Lambda_{p, q}^{1+}(\xi$, $K_{1}, K_{2}$ ) with the following additional assumption: there exists a number $\beta>0$, for which the operator $\left(\bar{V}^{\beta} x\right)(t)$ acts continuously from the space $m_{p}$ to the space $\Lambda_{p, q}^{1+}\left(\xi, K_{1}, K_{2}\right)$.

Below we assume, for the sake of simplicity, that the operator $Q$ in the reference equation (15) is deterministic. More specifically, it means the following.

Assumption 11. The fundamental matrix $U(t)$ of the reference equation (15) is deterministic and satisfies the inequality $\|U(t)\| \leq \widehat{c}$, where $\widehat{c}>0$ and $t \geq 0$.

Assumption 12. The Cauchy operator of the reference equation (15) has the integral representation

$$
(W g)(t)=\int_{0}^{t} C(t, s) g(s) d Z(s) \quad(t \geq 0),
$$

where $C(t, s)$ is an $n \times n$-matrix defined on $G:=\{(t, s): t \in$ $[0, \infty), 0 \leq s \leq t\}$, which satisfies on $G$ the estimate $\|C(t, s)\| \leq \widetilde{c} \exp \{-\alpha \Delta v\}$, where $v(t)=\int_{0}^{t} \xi(\zeta) d \lambda(\zeta), \Delta v=$ $v(t)-v(s)$ for some $\alpha>0, \widetilde{c}>0$, and a scalar, nonnegative function $\xi$, defined on $[0, \infty)$ and integrable with respect to the measure generated by the function $\lambda$.

The next two results give sufficient conditions for ISS with respect to pairs of weighted spaces. The proofs can be found in [18] and [19], respectively.

Theorem 13. Let $1 \leq p<\infty, 2 p \leq q<\infty$ and let (4) and the reference equation (15) satisfy the following conditions:

(1) The operators $V, Q$ act continuously from $M_{2 p}$ to $\Lambda_{2 p, q}^{n}\left(\xi, K_{1}, K_{2}\right)$.

(2) The reference equation (15) satisfies Assumptions 11 and 12.
(3) The operator $V$ satisfies the $\Delta$-condition from Definition 10.

If now the operator $I-\Theta_{l}: M_{2 p} \rightarrow M_{2 p}$ is continuously invertible, then (4) is ISS with respect to the pair $\left(M_{2 p}^{\gamma}\right.$, $\left.\left(\Lambda_{2 p, q}^{n}\left(\xi, K_{1}, K_{2}\right)\right)^{\gamma}\right)$ for some $\beta>0$.

Theorem 14. Let $1 \leq p<\infty$ and let (4) and the reference equation (15) satisfy the following conditions:

(1) The operators $V, Q$ act continuously from $M_{2 p}$ to $\Lambda_{2 p, \infty}^{n}\left(\xi, K_{1}, K_{2}\right)$.

(2) The reference equation (15) satisfies Assumptions 11 and 12.

(3) The operator $V$ satisfies the $\Delta$-condition from Definition 10.

If now the operator $I-\Theta_{r}: \Lambda_{2 p, \infty}^{n}\left(\xi, K_{1}, K_{2}\right) \rightarrow$ $\Lambda_{2 p, \infty}^{n}\left(\xi, K_{1}, K_{2}\right)$ has a bounded inverse, then (4) is ISS with respect to the pair $\left(M_{2 p}^{\gamma},\left(\Lambda_{2 p, \infty}^{n}\left(\xi, K_{1}, K_{2}\right)\right)^{\gamma}\right)$ for some $\beta>0$.

Remark 15. In the last two theorems it is required that the operator $V$ satisfies the $\Delta$-condition. This is always true if this operator comes from an ordinary stochastic differential equation. In the case of delay equations, the operator $V$ typically satisfies the $\Delta$-condition if the delay is bounded, but the latter assumption can be essentially generalized. This problem is studied in more detail in $[3,11-14,30,31]$ and in some other papers.

\section{Lyapunov Stability of Linear Hereditary Itô Equations}

Stability analysis of stochastic differential equations including delays and impulses, which is based on the Lyapunov second (direct) method and its generalizations, is technically difficult and, in some cases, probably, even impossible. In this and in the next section we concentrate on such examples of stochastic hereditary equations, where, as we show, our technique based on ISS and subsequent regularization is directly applicable.

We restrict ourselves to the case of Itô equations, which are driven by the standard Brownian motion [27], although the results presented below can be generalized to hereditary equations driven by semimartingales.

First of all, we notice that the stochastic process $Z(t)=$ $\left(t, \mathscr{B}_{2}(t), \ldots, \mathscr{B}_{m}(t)\right)$, where $\mathscr{B}_{i}, i=2, \ldots, m$, are the standard, mutually independent Brownian motions, is an $m$-dimensional semimartingale $[27,29]$, so that the theory presented in Sections 3 and 4 is directly applicable to Itô equations.

Given a number $1 \leq p<\infty$, the following inequality [27, Ineq. (3.1)] is essential for estimates of the operator norms in the spaces of stochastic processes:

$$
E\left|\int_{a}^{b} \zeta(t) d \mathscr{B}(t)\right|^{2 p} \leq c_{p} E\left(\int_{a}^{b}|\zeta(t)|^{2}(t) d t\right)^{p}
$$


where $\mathscr{B}$ is a scalar standard Brownian motion, $a, b$ are arbitrary positive numbers, $\zeta$ is an arbitrary measurable and $\left(\mathscr{F}_{t}\right)_{t>0}$-adapted (i.e., $\zeta(t)$ is $\left(\mathscr{F}_{t}\right)$-measurable for any $t \geq$ $0)$ scalar stochastic process on $[a, b]$, and $c_{p}$ is a universal constant which only depends on $p$. Note that $c_{1}=1$, while for $p>1$ the estimates for $c_{p}$ can be found in the literature (see, e.g., [27] or [32, page 40]), where $p$ should be replaced with $2 p$. Similar estimates for general semimartingales (the Burkholder-Davis-Gundy inequalities) are also available and can be used to estimate operator norms in the $W$-method, but we do not use these norms in the present paper referring the reader to the cited publications.

Our first example is a scalar Itô equation with infinite delay. The proof of this result can be found in [15].

Theorem 16. The zero solution of the equation

$$
\begin{aligned}
d x(t)= & \left(a \xi(t) x(t)+b \xi(t) x\left(\frac{t}{\tau_{0}}\right)\right) d t \\
& +c \sqrt{\xi(t) x}\left(\frac{t}{\tau_{1}}\right) d \mathscr{B}(t) \quad(t \geq 0),
\end{aligned}
$$

where $\xi(t)=I_{[0, r]}(t)+t I_{[r, \infty]}(t), t \geq 0\left(I_{A}(t)\right.$ is the indicator of $A), \mathscr{B}(t)$ is the standard scalar Brownian motion, and $a, b$, $c, \tau_{0}, \tau_{1}, r$ are real numbers $\left(\tau_{0}>1, \tau_{1}>1\right)$, is asymptotically $2 p$-stable (in $x_{0}$, as $\varphi$ is not needed) if there exists $\alpha>0$ for which

$$
\begin{aligned}
\mid a+b & +\alpha\left|+c_{p}\right| c \mid \sqrt{0,5 \alpha}+\left(|a b|+b^{2}\right) \delta_{0} \\
& +c_{p}|b c| \sqrt{\delta_{0}}<\alpha,
\end{aligned}
$$

where $\delta_{0}=\max \left\{\log \tau_{0},\left(1-\tau_{0}^{-1}\right) r\right\}$.

Our second example is an impulsive Itô equation with discrete, nonconstant delays:

$$
\begin{aligned}
d x(t)= & \sum_{j=0}^{m_{1}} A_{1 j}(t) x\left(h_{1 j}(t)\right) d t \\
& +\sum_{i=2}^{m} \sum_{j=0}^{m_{i}} A_{i j}(t) x\left(h_{i j}(t)\right) d \mathscr{B}_{i}(t) \\
x(\nu)= & \varphi(\nu) \quad(\nu<0), \\
x\left(\mu_{j}\right)= & A_{j} x\left(\mu_{j}-0\right) \quad(j=1,2,3, \ldots \text { a.s. }),
\end{aligned}
$$

where $\mu_{j}, A_{j}, j=1,2,3, \ldots$, are real numbers such that $0=\mu_{0}<\mu_{1}<\mu_{2}<\cdots, \lim _{j \rightarrow \infty} \mu_{j}=\infty ; \varphi$ is a stochastic process, which is independent of the standard, independent Brownian motions $\mathscr{B}_{i}, i=2, \ldots, m$, and which has a.s. essentially bounded trajectories; $A_{i j}, i=1, \ldots, m, j=$ $0, \ldots, m_{i}$, are $\mathscr{F}_{t}$-adapted $n \times n$-matrices, where $A_{1 j}, j=$ $0, \ldots, m_{1}$, are a.s. locally integrable and $A_{i j}, i=2, \ldots, m, j=$ $0, \ldots, m_{i}$, are square locally integrable with respect to the Lebesgue measure $\mu ; h_{i j}, i=1, \ldots, m, j=0, \ldots, m_{i}$, are
Borel measurable functions satisfying $h_{i j}(t) \leq t$ for $t \in$ $[0, \infty) \mu$-almost everywhere on $[0,+\infty)$.

Notice that system (38), (39a), and (39b) is a particular case of the general system (1) and (2a) considered in Section 1. Stability analysis can be therefore performed by our method with a proper choice of the reference equation.

Below we assume that $A_{i j}, h_{i j}, i=1, \ldots, m, j=$ $0, \ldots, m_{i}$, satisfy the following conditions:

(1) $\left\|A_{1 j}(t)\right\| \leq a_{1 j}(t) P \times \mu$-almost everywhere, where $a_{1 j}$ are locally $\mu$-integrable functions for $j=0, \ldots, m_{1}$;

(2) $\left\|A_{i j}(t)\right\| \leq a_{i j}(t) P \times \mu$-almost everywhere, where $a_{i j}$ are locally $\mu$-integrable functions for $i=2, \ldots, m, j=$ $0, \ldots, m_{i}$

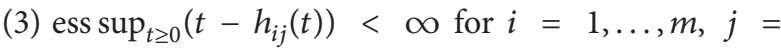
$0, \ldots, m_{i}$.

Remark 17. If in (39b) $A_{j}=1$ for $j=1,2,3, \ldots$, then (38), (39a), and (39b) becomes an Itô equation with delays; in this case condition (39b) can be omitted. If in (38) $h_{i j}(t)=t$ for $t \epsilon$ $[0, \infty) \mu$-almost everywhere for $i=1, \ldots, m, j=0, \ldots, m_{i}$, then (38) and (39b) becomes an Itô equation with impulses; in this case condition (39a) can be omitted.

Below $h^{T}(t)$ stands for the function on $[T, \infty)$, defined via the function $h(t)(t \in[T, \infty))$ by

$$
h^{T}(t)= \begin{cases}h(t), & \text { if } h(t) \geq T \\ T, & \text { if } h(t)<T .\end{cases}
$$

Let us formulate a result, the proof of which can be found in [22].

Theorem 18. Assume that $1 \leq p<\infty$ and that there exist a subset $I \subset\left\{0, \ldots, m_{1}\right\}$, the numbers $A, \rho, \sigma, \alpha, \gamma_{i}, i=1,2$, and $T \in[0, \infty)$ such that $\left|A_{j}\right| \leq A, \rho \leq \mu_{j+1}-\mu_{j} \leq \sigma$ for $j=k_{T}, k_{T}+1, \ldots$,

$$
\begin{aligned}
& \left\|\exp \left\{\int_{s}^{t} \sum_{k \in I} A_{1 k}(\tau) d \tau\right\}\right\| \leq \exp \{-\alpha(t-s)\}, \\
& A \exp \{-\alpha \rho\}<1, \\
& \sum_{k \in I} a_{1 k}(t)\left[\sum_{j=0}^{m_{1}} \int_{h_{1 k}^{T}(t)}^{t} a_{1 j}(s) d s\right. \\
& \left.\quad+c_{p} \sum_{i=2}^{m} \sum_{j=0}^{m_{i}}\left(\int_{h_{1 k}^{T}(t)}^{t}\left(a_{i j}(s)\right)^{2} d s\right)^{0.5}\right]+\sum_{k \bar{\epsilon} I} a_{1 k}(t) \\
& \quad \leq \gamma_{1}
\end{aligned}
$$


fort $\geq T \mu$-almost everywhere, $\sum_{i=2}^{m} \sum_{j=0}^{m_{i}}\left(\operatorname{ess}_{\sup } \operatorname{suT}_{t \geq T}\left(a_{i j}(t)\right)^{2}\right)^{0.5}$ $=\gamma_{2}$ and, finally,

$$
\begin{aligned}
& \frac{\max \{1, A\}(1-\exp \{-\alpha \sigma\})}{\alpha(1-\exp \{-\alpha \rho\} A)} \gamma_{1} \\
& \quad+c_{p} \gamma_{2}\left[\frac{\max \left\{1, A^{2}\right\}(1-\exp \{-2 \alpha \sigma\})}{2 \alpha(1-\exp \{-2 \alpha \rho\} A)}\right]^{1 / 2}<1 .
\end{aligned}
$$

Then the zero solution of (38) and (39b) is exponentially $2 p$ stable.

In the paper [22] several corollaries of this theorem are listed, which can be used to obtain more explicit conditions of the exponential $2 p$-stability $(1 \leq p<\infty)$ of (38) and (39b) with respect to the initial data.

In the next theorem we study asymptotic stability of (38) and (39b) in the case of unbounded delays. We assume therefore that $h_{i j}(t)=t / h_{i j}$ for $t \in[0,+\infty)$, where $h_{i j}$ are real numbers such that $h_{i j} \geq 1$ for $i=1, \ldots, m, j=0, \ldots, m_{i}$. According to Remark 17 condition (39a) is excessive in this case.

To be able to formulate the theorem we need some more notation. Let $\gamma(t)=t^{\beta}$, where $\beta$ is a positive number, $\widehat{h}_{i j}(t)=$ $t / h_{i j}$ for $i=1, \ldots, m, j=0, \ldots, m_{i}$, and $\hat{h}_{i j}^{T}(t), \chi_{\widehat{h}_{i j}}^{T}(t)$ are functions on $[T, \infty)$ defined as

$$
\begin{aligned}
& \widehat{h}_{i j}^{T}(t)= \begin{cases}\widehat{h}_{i j}(t), & \text { if } \widehat{h}_{i j}(t) \geq T, \\
T, & \text { if } \widehat{h}_{i j}(t)<T,\end{cases} \\
& \chi_{\widehat{h}_{i j}^{T}}^{T}(t)= \begin{cases}1, & \text { if } \widehat{h}_{i j}(t) \geq T, \\
0, & \text { if } \widehat{h}_{i j}(t)<T,\end{cases}
\end{aligned}
$$

and $\widehat{A}_{i j}(t)=A_{i j}(t) \chi_{\widehat{h}_{i j}}^{T}(t)$ for $i=1, \ldots, m, j=0, \ldots, m_{i}$; put also $\xi(t)=1 / t(t \geq T)$.

The following theorem was proved in [23].

Theorem 19. Let $1 \leq p<\infty$ and for system (38) and $(39 b)$ there exist a subset $I \subset\left\{0, \ldots, m_{1}\right\}$, positive numbers $A, \rho, \sigma, \alpha, \gamma_{i}, i=1,2$, and $T \in[0, \infty)$ such that $a_{1 j} / \xi \in$ $L_{\infty}^{T}\left(j=0, \ldots, m_{1}\right), a_{i j} / \sqrt{\xi} \in L_{\infty}^{T}(i=2, \ldots, m, j=$ $\left.0, \ldots, m_{i}\right),\left|A_{j}\right| \leq A$, and $\rho \leq \ln \left(\mu_{j+1} / \mu_{j}\right) \leq \sigma\left(j=k_{T}, k_{T}+\right.$ $1, \ldots)$. Assume also that $A_{1 k}, k \in I$, are nonrandom functions and

$$
\begin{aligned}
& \left\|\exp \left\{\int_{s}^{t} \sum_{k \in I} \widehat{A}_{1 k}(\tau) d \tau\right\}\right\| \leq \exp \left\{-\alpha \ln \left(\frac{t}{s}\right)\right\}, \\
& A \exp \{-\alpha \rho\}<1,
\end{aligned}
$$

$$
\begin{aligned}
& \sum_{k \in I} t a_{1 k}(t)\left(\sum_{j=0}^{m_{1}} \int_{\hat{h}_{1 k}^{T}(t)}^{t} a_{1 j}(s) d s\right. \\
& \left.\quad+c_{p} \sum_{i=2}^{m} \sum_{j=0}^{m_{i}}\left(\int_{\hat{h}_{1 k}^{T}(t)}^{t}\left(a_{i j}(s)\right)^{2} d s\right)^{0.5}\right)+\sum_{k \in \bar{I}} t a_{1 k}(t) \\
& \leq \gamma_{1}
\end{aligned}
$$

for $t \geq T \mu$-almost everywhere. Finally, let inequality (42) be fulfilled for this $\gamma_{1}$ and

$$
\gamma_{2}=\sum_{i=2}^{m} \sum_{j=0}^{m_{i}} \operatorname{ess} \sup _{t \geq T}\left(\sqrt{t} a_{i j}(t)\right) \text {. }
$$

Then the zero solution of (38) and (39b) is asymptotically $2 p$ stable.

In [23] several corollaries of this theorem are given, which provide more explicit conditions of the asymptotic $2 p$ stability $(1 \leq p<\infty)$ of the impulsive equations of the form (38) and (39b).

\section{Itô Type Linear Functional Difference Equations}

Stochastic difference equations were truly defined in [33]. Stochastic functional difference equations were introduced in [34] and studied further in [35]. Analysis of Lyapunov stability for stochastic difference equations is a challenging mathematical problem which has attracted attention of many researchers but has not yet been comprehensively studied. Some theorems on stability of ordinary stochastic difference equations can be, for example, found in [36-39], while results on stability of stochastic functional difference equations are summarized in the monograph [40]. As in the case of differential equations, stochastic versions of Lyapunov's classical methods can be applied to difference equations, too. These methods work in many situations, yet some important classes of equations seem to be insufficiently studied, which may be due to several technical restrictions one has to put on Lyapunov functions and especially functionals, in the stochastic case. In this section we apply our method instead and show that it is efficient in many situations.

In the sequel $N$ is the set of all natural numbers, $N_{+}=$ $\{0\} \cup N$, and the variable $s$ is always assumed to belong to $N_{+}$; that is, $s=0,1,2, \ldots$.

Below we consider the following stochastic difference equations:

(a) The Linear Ordinary Difference Itô Equation

$$
\begin{aligned}
x(s+1) & \\
= & x(s)+A_{1}(s) x(s) h \\
& +\sum_{i=2}^{m} A_{i}(s) x(j)\left(\mathscr{B}_{i}((s+1) h)-\mathscr{B}_{i}(s h)\right)
\end{aligned}
$$


where $x(s)$ is a $\mathscr{F}_{s}$-measurable, $n$-dimensional random variable for any $s \in N_{+}, h$ is a positive number, and $A_{i}(s)$ is an $n \times n$-matrix, whose entries are $\mathscr{F}_{s}$-measurable random variables for any $i=1, \ldots, m, s \in N_{+}$.

(b) The Linear Difference Itô Equation with Delay

$$
\begin{aligned}
& x(s+1)=x(s)+\sum_{j=-\infty}^{s} A_{1}^{2}(s, j) x(j) h \\
& \quad+\sum_{i=2}^{m} \sum_{j=-\infty}^{s} A_{i}^{2}(s, j) x(j)\left(\mathscr{B}_{i}((s+1) h)-\mathscr{B}_{i}(s h)\right) \\
& x(j)=\varphi(j) \quad\left(j \in N_{+}\right),
\end{aligned}
$$

where $x(s)$ is a $\mathscr{F}_{s}$-measurable, $n$-dimensional random variable for any $s \in N_{+}$, $h$ is a positive number, and $A_{i}^{2}(s, j)$ is an $n \times n$-matrix, whose entries are $\mathscr{F}_{s}$-measurable random variables for any $s \in N_{+}, j=-\infty, \ldots, s, i=1, \ldots, m, \varphi(j)$ $(j<0)$ is a $\mathscr{F}_{0}$-measurable random variable.

Note that (46) is a particular case of (47). Below we therefore formulate the definitions and results in terms of (47), only.

A solution of (47) is a sequence of $n$-dimensional and $\mathscr{F}_{s}$-measurable random variables $x(s) \quad(s=0, \pm 1, \pm 2, \ldots)$, which $P$-almost surely satisfies (47) and (48a) and a given initial condition $x(0)=x_{0} \in k^{n}$. Note that, for any $\mathscr{F}_{0^{-}}$ measurable initial value $x_{0}$, the solution of (47) always exists, and it is unique up to the natural $P$-equivalence. Moreover, this solution is $\mathscr{F}_{s}$-adapted for all $s=0, \pm 1, \pm 2, \ldots$.

For given $1 \leq p<\infty, h>0$, the number $c_{p}^{h}$ is the universal constant for which the following inequality is satisfied:

$$
E\left|\int_{t}^{t+h} \phi(s) d \mathscr{B}(s)\right|^{2 p} \leq c_{p}^{h} E \int_{t}^{t+h}|\phi(s)|^{2 p} d s .
$$

The inequality should be valid for any $t \geq 0$, any $\mathscr{F}_{t}$-adapted stochastic process $\phi$, and a standard scalar Wiener process $\mathscr{B}$. In [32, page 39], these constants are defined (up to a change of the notation) as $c_{p}^{h}=p^{p}(2 p-1)^{p} h^{p-1}$ for $p>1$ and $c_{1}=1$ for $p=1$. The Burkholder-Davis-Gundy inequalities give the estimates which are independent of $h$ (see, e.g., [32, page 40], where $p$ should be replaced by $2 p$ ).

The first result is about asymptotic stability of the ordinary difference equation (46).

Theorem 20. Assume that $A_{i}(s)=a_{i}, i=1, \ldots, m$, for $s \in$ $N_{+}$.

If now

$$
c_{p}^{h} \sum_{i=2}^{m}\left|a_{i}\right|<-a_{1} h^{1 / 2}, \quad-1<a_{1} h<0,
$$

then the zero solution of (46) is asymptotically $2 p$-stable with respect to initial data.
The second result applies to the vector equation (47). However, it does not guarantee asymptotic stability.

Theorem 21. Assume that there exist positive numbers $a_{i}(s, j), i=1, \ldots, n, s \in N_{+}, j=-\infty, \ldots, s$, such that the coefficients in (13) satisfy

$$
\begin{aligned}
& \left\|A_{i}(s, j)\right\| \leq a_{i}(s, j), \\
& \quad i=1, \ldots, m, s \in N_{+}, j=-\infty, \ldots, s
\end{aligned}
$$

P-almost surely,

$$
\begin{aligned}
& \sum_{\tau=0}^{\infty} \sum_{j=-\infty}^{-1} a_{i}(\tau, j)<\infty \quad(i=1, \ldots, m), \\
& \bar{c} \stackrel{\text { def }}{=} \sum_{\tau=0}^{\infty}\left(\sum_{j=0}^{\tau} a_{1}(\tau, j) h+c_{p}^{h} \sum_{i=2}^{m} \sum_{j=0}^{\tau} a_{i}(\tau, j) h^{1 / 2}\right)<1 .
\end{aligned}
$$

Then the zero solution of (47) is $2 p$-stable with respect to the initial data.

The Idea of the Proof. The proofs of the theorems are based on the discrete analogue of the $W$-method and can be found in $[24,25]$. Below we present a sketch of the proof.

First of all, let us represent (46) and (47) in the form of the following discrete operator equation:

$$
x(s+1)=x(s)+[(V x)(s)+f(s)] Y(s) \quad\left(s \in N_{+}\right),
$$

where

$$
\begin{aligned}
& (V x)(s)=\left(\sum_{j=0}^{s} A_{1}(s, j) x(j), \sum_{j=0}^{s} A_{2}(s, j) x(j), \ldots,\right. \\
& \left.\sum_{j=0}^{s} A_{m}(s, j) x(j)\right) \quad\left(s \in N_{+}\right), \\
& f=\left(\sum_{j=-\infty}^{-1} A_{1}^{2}(\cdot, j) \varphi(j), \ldots, \sum_{j=-\infty}^{-1} A_{m}^{2}(\cdot, j) \varphi(j)\right) \\
& Y(s)=\left(h,\left(\mathscr{B}_{2}((s+1) h)-\mathscr{B}_{2}(s h)\right), \ldots,\right. \\
& \left.\left(\mathscr{B}_{m}((s+1) h)-\mathscr{B}_{m}(s h)\right)\right) \quad\left(s \in N_{+}\right) .
\end{aligned}
$$

Then, we observe that (53) is a particular case of the general functional differential equation (4) if we put $Z(t)=$ $\sum_{s>t, s \in N} Y(s) I(t-s)$, where

$$
I(t)= \begin{cases}t, & \text { if } t \geq 0 \\ 0, & \text { if } t<0\end{cases}
$$

By this, the theory presented in Sections 3 and 4 is directly applicable. For the sake of completeness, let us describe the discrete analogues of the spaces $D^{n}$ and $L^{n}(Z)$ :

(1) $d^{n}$ is the linear space of all possible solutions of the difference equation (53). 
(2) $l^{n}$ is the linear space of all sequences of $m \times n$-matrices $H(s)\left(s \in N_{+}\right)$, with the entries being $\mathscr{F}_{s}$-measurable random variables.

It is easy to see that $V$ is a linear operator from $d^{n}$ to $l^{n}$.

The normed subspace $B$ can be now chosen in the way described in Section 4. We omit here the technicalities referring the reader to $[24,25]$.

\section{Conclusion}

In the present paper we considered the property of $p$-stability $(1 \leq p<\infty)$ for various linear stochastic equations. We showed how to obtain efficient stability results formulated in terms of the parameters of the equations in question. However, let us stress that the central results of the paper are also valid for other kinds of stochastic stability, for example, stability in probability and almost sure stability (see, e.g., [13]). The major technical problem arising in analysis of these types of stability is mostly due to the fact that the corresponding spaces of stochastic processes do not admit norms, which makes the analysis of invertibility of the associated linear operators more difficult and less efficient.

Moreover, the method can also be applied to analysis of other asymptotic properties of stochastic equations and not only to the Lyapunov stability. For instance, in $[13,14,16,41]$ the problem of partial Lyapunov stability was addressed.

Unlike the Lyapunov-Krasovskii-Razumikhin method, which is based on the existence of a suitable Lyapunov function (or the Lyapunov-Krasovskii functional in the delay case), our method is based on the ISS property of the equation and requires a suitable auxiliary (reference) equation which is used to regularize the equation under consideration. The crucial point is then to check solvability of a regularized equation in a carefully chosen space of stochastic processes. This yields ISS and thus the desirable asymptotic property.

Several techniques of constructing reference equations have been worked out in the papers cited above. Normally, such an equation is chosen to be dependent on a parameter, which ensures a certain flexibility. This approach has led to several new and interesting results, many of which seem to be difficult (impossible?) to obtain by other methods. One of such examples, equations with random delays, is studied in [17]. It is also worth mentioning that our techniques allow for studying Lyapunov stability with respect to random initial data (see Definition 1), which is rarely possible when using Lyapunov function(al)s.

Another crucial idea, which is of great importance in the stability theory, is the Bohl-Perron principle, which states that asymptotic and exponential stability in rather general cases can be deduced from invertibility of the operators in nonweighted spaces. Technically, it is much easier to deal with the latter spaces rather than the spaces with a nontrivial weight. It turned out to be possible to formulate the Bohl-Perron principle in terms of the verifiable $\Delta$-condition. Roughly speaking, this condition says that the delays in the system should not be unbounded, although many delay equations with unbounded delays also satisfy the $\Delta$-condition.

Finally, we remark that although the method was originally designed for stochastic linear equations, several classes of nonlinear stochastic functional differential equations can also be investigated similarly; see $[12,13,16]$ for some results. In [26] the mean square input-to-state stability was introduced to study neural networks. The technique is rather promising and might be particularly efficient in several important applied problems, for example, in the synchronization theory for networks with random switching [42].

\section{Competing Interests}

The authors declare that they have no competing interests.

\section{Acknowledgments}

Both authors have been partially supported by the Norwegian Research Council, Grant no. 239070.

\section{References}

[1] E. D. Sontag, "Input to state stability: basic concepts and results," in Nonlinear and Optimal Control Theory, Lecture Notes in Mathematics, pp. 163-220, 2008.

[2] N. V. Azbelev and P. M. Simonov, Stability of Differential Equations with Aftereffect, Taylor and Francis, London, UK, 2002.

[3] R. I. Kadiev and A. Ponosov, "Stability of stochastic functionaldifferential equations under constantly acting perturbations," Differential Equations, vol. 28, no. 2, pp. 198-207, 1992.

[4] L. M. Berezansky, "Development of N. V. Azbelev's W-method for stability problems for solutions of linear functional differential equations," Differential Equations, vol. 22, pp. 521-529, 1986.

[5] N. V. Azbelev, L. M. Berezansky, P. M. Simonov, and A. V. Chistyakov, "Stability of linear systems with time-lag," Journal of Differential Equations, vol. 23, pp. 493-500, 1987.

[6] N. V. Azbelev, L. M. Berezansky, P. M. Simonov, and A. V. Chistyakov, "Stability of linear systems with time-lag," Differential Equations, vol. 27, pp. 383-388, 1165-1172, 1991.

[7] N. V. Azbelev, L. M. Berezansky, P. M. Simonov, and A. V. Chistyakov, "Stability of linear systems with time-lag," Differential Equations, vol. 29, pp. 153-160, 1993.

[8] A. Anokhin, L. Berezansky, and E. Braverman, "Exponential stability of linear delay impulsive differential equations," Journal of Mathematical Analysis and Applications, vol. 193, no. 3, pp. 923-941, 1995.

[9] L. Berezansky and E. Braverman, "On exponential dichotomy, Bohl-Perron type theorems and stability of difference equations," Journal of Mathematical Analysis and Applications, vol. 304, no. 2, pp. 511-530, 2005.

[10] L. Berezansky and E. Braverman, "On exponential stability of linear differential equations with several delays," Journal of Mathematical Analysis and Applications, vol. 324, no. 2, pp. 1336-1355, 2006.

[11] R. I. Kadiev, "Sufficient conditions for stability of stochastic systems," Differential Equations, vol. 33, no. 3, pp. 423-424, 1997. 
[12] R. I. Kadiev, "On the stability of stochastic functional-differential equations with respect to the first approximation," Russian Mathematics, vol. 43, no. 10, pp. 1-6, 1999.

[13] R. I. Kadiev, Stability of solutions of stochastic functional differential equations [Habilitation thesis], Jekaterinburg State University, Jekaterinburg, Russia, 2000 (Russian).

[14] R. I. Kadiev, "Sufficient conditions for stability with respect to part of the variables of linear stochastic systems with aftereffect," Russian Mathematics, vol. 6, p. 7579, 2000.

[15] R. I. Kadiev, "Asymptotic stability of itô differential systems with retarded argument," Differential Equations, vol. 36, no. 2, pp. 187-192, 2000.

[16] R. I. Kadiev, "Stability of solutions with respect to part of the variables of stochastic functional-differential equations with respect to the first approximation," Russian Mathematics, vol. 5, p. 3035, 2001.

[17] R. I. Kadiev, "Stability of solutions of stochastic differential equations with random delays," Differential Equations, vol. 40, no. 2, pp. 276-281, 2004.

[18] R. Kadiev and A. Ponosov, "Stability of stochastic functional differential equations and the Azbelev transform," Electronic Journal of Differential Equations, vol. 92, pp. 1-36, 2004.

[19] R. I. Kadiev and A. Ponosov, "Relations between stability and admissibility for stochastic linear functional differential equations," Functional Differential Equations, vol. 12, pp. 117-141, 2005.

[20] R. I. Kadiev and A. Ponosov, "Exponential stability of linear stochastic differential equations with bounded delay and the Azbelev transform," Electronic Journal of Qualitative Theory of Differential Equations, vol. 23, pp. 1-14, 2008.

[21] R. I. Kadiev and A. Ponosov, "Development of N. V. Azbelev's ideas in the stability theory for stochastic functional differential equations," Functional Differential Equations, vol. 15, pp. 195213, 2008.

[22] R. I. Kadiev and A. Ponosov, "Stability of linear impulsive Itô differential equations with bounded delays," Differential Equations, vol. 46, no. 4, pp. 489-501, 2010.

[23] R. I. Kadiev and A. Ponosov, "Stability of impulsive stochastic differential linear functional equations with linear delays," Journal of Abstract Differential Equations and Applications, vol. 2, no. 2, pp. 7-25, 2012.

[24] R. Kadiev and A. Ponosov, "The $W$-transform in stability analysis for stochastic linear functional difference equations," Journal of Mathematical Analysis and Applications, vol. 389, no. 2, pp. 1239-1250, 2012.

[25] R. Kadiev and A. Ponosov, "Exponential stability of Itô-type linear functional difference equations," Computers and Mathematics with Applications, vol. 66, no. 11, pp. 2295-2306, 2013.

[26] Q. Zhu and J. Cao, "Mean-square exponential input-to-state stability of stochastic delayed neural networks," Neurocomputing, vol. 131, pp. 157-163, 2014.

[27] R. Sh. Liptser and A. N. Shirjaev, Theory of Martingales, Kluwer Academic, Dordrecht, The Netherlands, 1989.

[28] R. I. Kadiev, "Existence and uniqueness of the solution of the Cauchy problem for functional-differential equations with respect to a semimartingale," Izvestiya Vysshikh Uchebnykh Zavedenii. Matematika, no. 10, pp. 35-39, 1995.

[29] J. Jacod, "Integrales stochastiques par rapport a une semimartingale vectorielle et change-ments de filtration," in Séminaire de Probabilités XIV 1978/79, vol. 784 of Lecture Notes in Mathematics, pp. 161-172, Springer, 1980.
[30] R. I. Kadiev, "Sufficient conditions for stability of stochastic systems with aftereefect," Differential Equations, vol. 30, no. 4, pp. 555-564, 1994.

[31] R. I. Kadiev, “The admissibility of pairs of spaces with respect to some of the variables for linear stochastic functionaldifferential equations," Izvestiya Vysshikh Uchebnykh Zavedenii. Matematika, vol. 5, pp. 13-22, 1994.

[32] X. Mao, Stochastic Differential Equations \& Applications, Horwood, Chichester, UK, 1997.

[33] D. S. Clark, "A stochastic difference equation," Journal of Differential Equations, vol. 40, no. 1, pp. 71-93, 1981.

[34] X. Mao, "Numerical solutions of stochastic functional differential equations," LMS Journal of Computation and Mathematics, vol. 6, pp. 141-161, 2003.

[35] F. Wu and X. Mao, "Numerical solutions of neutral stochastic functional differential equations," SIAM Journal on Numerical Analysis, vol. 46, no. 4, pp. 1821-1841, 2008.

[36] C. T. Baker and E. Buckwar, "Exponential stability in $p$-th mean of solutions, and of convergent Euler-type solutions, of stochastic delay differential equations," Journal of Computational and Applied Mathematics, vol. 184, no. 2, pp. 404-427, 2005.

[37] D. J. Higham, X. Mao, and C. Yuan, "Almost sure and moment exponential stability in the numerical simulation of stochastic differential equations," SIAM Journal on Numerical Analysis, vol. 45, no. 2, pp. 592-609, 2007.

[38] B. Paternoster and L. Shaikhet, "About stability of nonlinear stochastic difference equations," Applied Mathematics Letters, vol. 13, no. 5, pp. 27-32, 2000.

[39] A. Rodkina, "On asymptotic behaviour of solutions of stochastic difference equations," Nonlinear Analysis: Theory, Methods \& Applications, vol. 47, no. 7, pp. 4719-4730, 2001, Proceedings of the 3rd World Congress of Nonlinear Analysts, Part 7.

[40] L. Shaikhet, Lyapunov Functionals and Stability of Stochastic Difference Equations, Springer, London, UK, 2011.

[41] R. I. Kadiev and A. Ponosov, "Partial stability of stochastic functional differential equations and the Azbelev transform," International Journal of Dynamics of Continuous, Discrete and Impulsive Systems (DCDIS), Series A: Mathematical Analysis, vol. 21, no. 1, pp. 1-35, 2014.

[42] L. Pan, J. Cao, and J. Hu, "Synchronization for complex networks with Markov switching via matrix measure approach," Applied Mathematical Modelling, vol. 39, no. 18, pp. 5636-5649, 2015. 


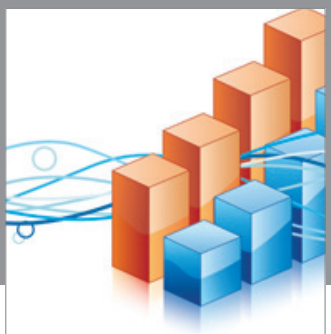

Advances in

Operations Research

vatem alat4

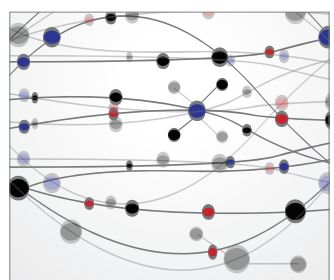

\section{The Scientific} World Journal
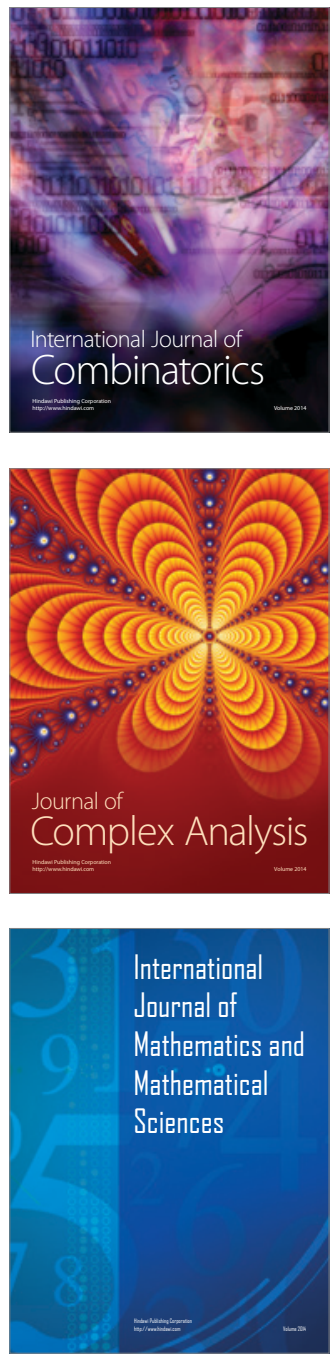
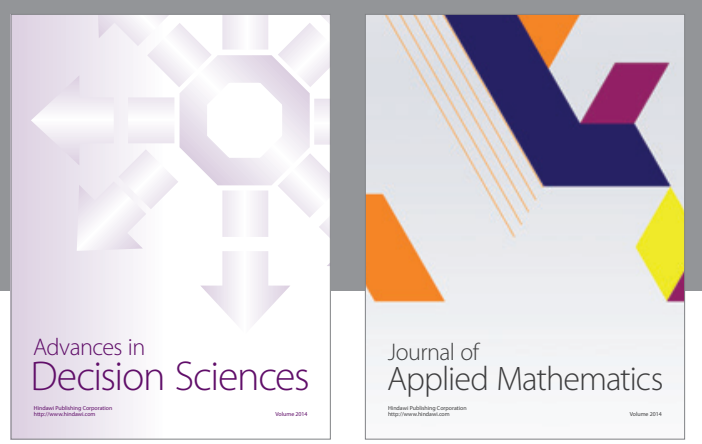

Algebra

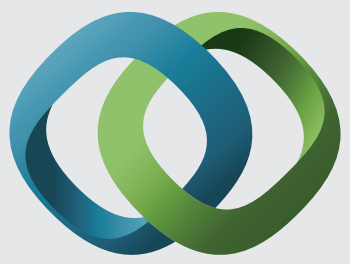

\section{Hindawi}

Submit your manuscripts at

http://www.hindawi.com
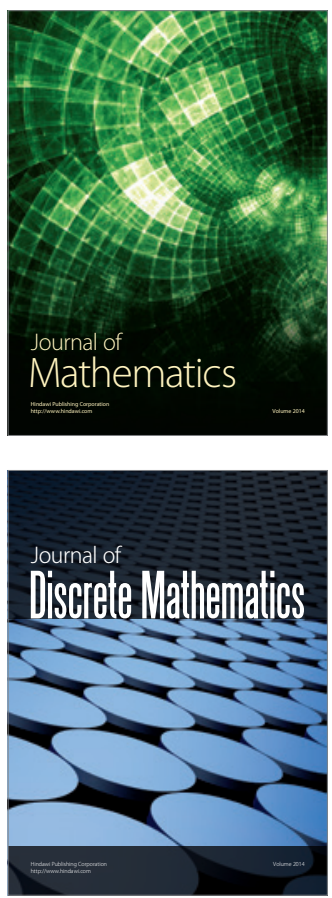

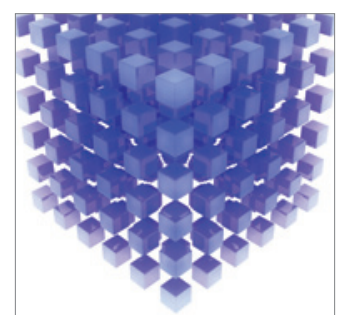

Mathematical Problems in Engineering
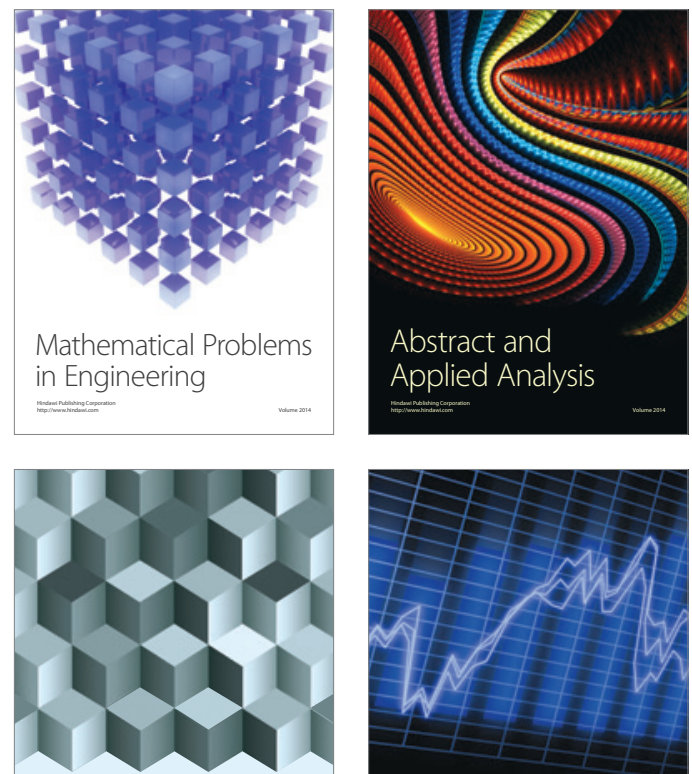

Journal of

Function Spaces

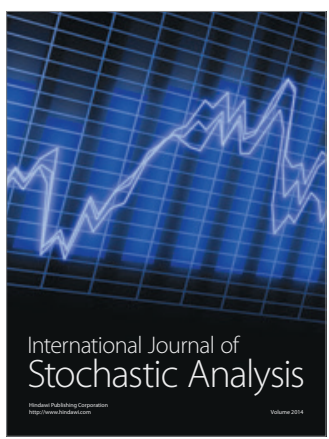

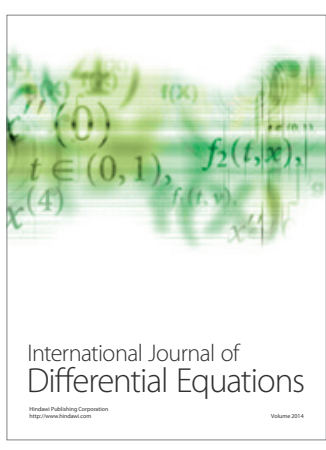
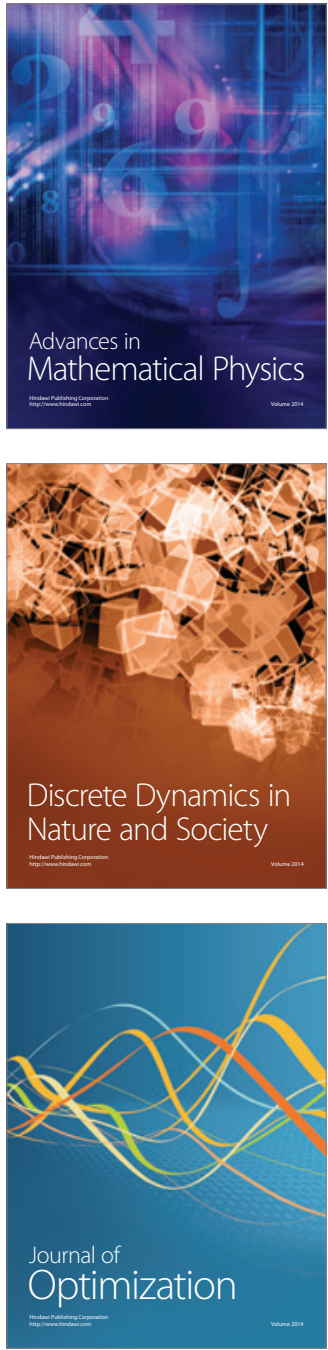